\title{
The Influence of DCs Loaded with Tumor Antigens on the Cytotoxic Response of MNC Culture Patients with Oncology
}

\author{
A.P. Cherkasov, J.N. Khantakova, S.A. Falaleeva, A.A. Khristin, \\ N.A. Kiryishina, V.V. Kozlov, E.V. Kulikova, V.V. Kurilin, \\ J.A. Lopatnikova, I.A. Obleukhova, S.V. Sennikov, J.A. Shevchenko, \\ S.V. Sidorov, A.V. Sokolov and A.E. Vitsin
}

\begin{abstract}
Currently, one of the most promising approaches for the treatment of oncological patients is the selective activation of T-cell antitumor immunity using immune cells. The generation of functionally active DCs in vitro constitutes a promising approach in the development of DC-based anticancer vaccines to mobilize patient defense systems, because their activation by tumor-specific antigens to induce cytotoxic responses, and their increased efficiency of antigen presentation to induce cytotoxic T lymphocytes (CTLs) via costimulatory molecules and cytokines can be controlled. The purpose of this study was to investigate the
\end{abstract}

A.P. Cherkasov $(\bowtie) \cdot$ A.A. Khristin · A.V. Sokolov · A.E. Vitsin

City Clinical Hospital no. 1, Pirogov, Russia

e-mail: cherkassov333@mail.ru

A.A. Khristin

e-mail: AAlex.Khristin@gmail.com

A.V. Sokolov

e-mail: sokolowav2003@mail.ru

A.E. Vitsin

e-mail: gella2009@yandex.ru

J.N. Khantakova - S.A. Falaleeva · E.V. Kulikova · V.V. Kurilin · J.A. Lopatnikova ·

I.A. Obleukhova · S.V. Sennikov · J.A. Shevchenko

Federal State Budgetary Scientific Institution "Research Institute of Fundamental and Clinical Immunology" Laboratory of Molecular Immunology, Novosibirsk, Russia e-mail: KhantJN@gmail.com

S.A. Falaleeva

e-mail: kolenteonok@mail.com

E.V. Kulikova

e-mail: homka-88@inbox.ru

V.V. Kurilin

e-mail: 2221910@ngs.ru

J.A. Lopatnikova

e-mail: lopatnikova_j_a@ngs.ru

(C) The Author(s) 2018

K.V. Anisimov et al. (eds.), Proceedings of the Scientific-Practical Conference

"Research and Development - 2016”, https://doi.org/10.1007/978-3-319-62870-7_56 
functional characteristics of peripheral blood DC subsets in colorectal cancer (CRR), breast cancer (BC), and non-small cell lung cancer (NSCLC) patients and the development of an antitumor cytotoxic response by mononuclear cells (MNCs) from patients using in vitro generated antigen-primed DCs.

Keywords Antigen-primed dendritic cells - Antitumor cytotoxic response Colorectal cancer $\cdot$ Breast cancer $\cdot$ Non-small cell lung cancer

\section{Introduction}

The generation of functionally active DCs in vitro constitutes a promising approach in the development of DC-based anticancer vaccines to mobilize patient defense systems, because their activation by tumor-specific antigens to induce cytotoxic responses, and their increased efficiency of antigen presentation to induce cytotoxic $\mathrm{T}$ lymphocytes (CTLs) via costimulatory molecules and cytokines can be controlled [5, p. 459; 1, p. 1]. DC-based anticancer vaccines are delivered using various methods, including the use of free peptides [10, p. 1868], tumor lysates [3, p. 2827], DNA [12, p. $1339 ; 8$, p. 122] or RNA vaccines [9, p. 1], as well as DCs primed with various tumor antigens [6, p. 57]. Because tumors have a heterogeneous structure, the surface of cells comprising the tumor bears an individual set of TAAs. The use of tumor lysate as a source of tumor immunogens has the potential advantage of stimulating a response against a variety of known and unknown TAAs typical of both a particular tumor type and a particular patient. This method enables the induction of a polyclonal immune response, stimulating both helper CD4+ and cytotoxic CD8+ immune responses, thereby reducing the risk of the tumor escaping immune surveillance [7, p. 139]. The use of a tumor lysate reduces the time and effort spent identifying and synthesizing immunodominant peptide epitopes, enabling DCs to process tumor antigens naturally.

I.A. Obleukhova

e-mail: obleukhova.irina@yandex.ru

S.V. Sennikov

e-mail: sennikovsv@gmail.com

J.A. Shevchenko

e-mail: shevja80@gmail.com

N.A. Kiryishina · V.V. Kozlov

Novosibirsk Regional Clinical Oncology Center, Novosibirsk, Russia

e-mail: natahamed@mail.ru

V.V. Kozlov

e-mail: vadimkozlov80@mail.ru

S.V. Sidorov

Department of Surgery, Novosibirsk State University, Novosibirsk Oblast, Russia e-mail: naf202@mail.ru 


\section{Materials and Methods}

Study subjects. Peripheral blood and tumor samples were obtained from:

- 44 patients aged 42-83 years (mean $=66.8$ years) with colorectal cancer at stages I-IV [I-II-30 patients $(68.2 \%)$, III-IV-14 patients $(31.8 \%)$ ], including 21 men $(47.7 \%)$, and 23 women $(52.3 \%)$. Colorectal adenocarcinoma was confirmed in all patients.

- 16 patients with the histologically verified diagnosis of non-small cell lung cancer (NSCLC) stage IIA, IIB, and IIIA as well as tumor biopsy material obtained during surgery (14 males, 2 females; mean age $60.7 \pm 1.5$ years).

- 20 patients aged 35-77 years (mean age, 57.9 years) with the histologically and immunohistochemically verified diagnosis breast cancer at stages I-II.

All patients provided their informed consent to participate in this study. Patient diagnostic and histology data were kindly provided by the medical institution (City Clinical Hospital No. 1 and Novosibirsk Regional Clinical Oncology Center, Novosibirsk, Russia), where patient examination and clinical surveillance (treatment) was performed.

DC preparation. Peripheral blood MNCs were isolated using standard Ficoll-Urografin density-gradient [2, p. 97]. Cells with enhanced adherence were isolated from the resulting MNC population using a short incubation period $(2 \mathrm{~h})$ in a $75-\mathrm{cm}^{2}$ culture flask, in $5 \% \mathrm{CO}_{2}$ at $37{ }^{\circ} \mathrm{C}$. Cells were grown in RPMI-1640 complete medium supplemented with $10 \%$ fetal bovine serum (FBS), $40 \mu \mathrm{g} / \mathrm{mL}$ gentamicin, $200 \mathrm{U} / \mathrm{mL}$ penicillin, $2 \mathrm{mM}$ L-glutamine, $5 \times 10^{-5} \mathrm{M}$ 2-mercaptoethanol, and $10 \mathrm{mM}$ HEPES. The adherent MNC fraction was cultured in 48 -well plates at $1 \times 10^{6}$ cells $/ \mathrm{mL}$ in $0.5 \mathrm{~mL}$ complete medium. The adherent MNC fraction was supplemented with $50 \mathrm{ng} / \mathrm{mL}$ rhGM-CSF and $100 \mathrm{ng} / \mathrm{mL}$ rhIL-4 to produce immature DCs through a 4-day incubation. To obtain tumor antigen-loaded DCs, immature DCs were supplemented with autologous tumor cell lysate at a concentration of $100 \mu \mathrm{g} / \mathrm{mL}$ after $48 \mathrm{~h}$ of incubation. To generate DCs transfected with tumor cell RNA, we performed magnetic transfection using Promokine reagents (Germany), according to the manufacturer protocol. The culture was then supplemented with rhTNF- $\alpha(25 \mathrm{ng} / \mathrm{mL})$ in fresh culture medium and an equivalent volume to generate mature cells over the following $24 \mathrm{~h}$. The fraction of non-adherent cells was maintained in a $75-\mathrm{cm}^{2}$ culture flask at $2 \times 10^{6}$ cells $/ \mathrm{mL}$ in RPMI 1640 complete medium until decantation.

Preparation of autologous tumor cells. A tumor sample was washed in RPMI-1640 with a doubled concentration of antibiotics. To obtain autologous tumor cells, a tumor fragment from each patient was crushed and left in $0.25 \%$ trypsin solution at $+4{ }^{\circ} \mathrm{C}$ overnight. Warm complete RPMI-1640 was used to inactivate the enzyme. The cell suspension was filtered to remove large aggregates, washed twice, and frozen in FBS with 10\% DMSO (Panreacsintesis, Spain). To obtain tumor cell lysates, a tumor fragment was mechanically homogenized and the resulting suspension was successively frozen at $-70{ }^{\circ} \mathrm{C}$ and thawed at $+37{ }^{\circ} \mathrm{C}$ 
through three cycles. Cells were pelleted by centrifugation and sterilized by passing through a $0.45-\mu \mathrm{m}$ filter. Total protein in the lysates was determined by calculating the $260 / 280 \mathrm{~nm}$ absorbance ratio using a NanoDrop device (Thermo Scientific, USA).

Co-culture of DC and MNC. Co-culture of DC and MNC was carried out in several parallel cultures for subsequent functional tests under uniform conditions. The concentration of non-adherent cultured MNC was $1 \times 10^{6}$ cells $/ \mathrm{ml}$, the DC: MNC ratio was 1:10, and rhIL-12 (10 ng/ml; PeproTech, USA) and rhIL-18 (100 ng/ml; MBL, USA) were applied to stimulate Th1-polarization. Mononuclear cells used to assess cytotoxicity against autologous tumor cells were cultured for 4 days in complete RPMI-1640 in the presence or absence of recombinant cytokines. Mononuclear cells used for assessing perforin levels were cultured in complete RPMI-1640 in the presence or absence of recombinant cytokines for 2 days; the cultures were subsequently washed of growth factors, and cultured for a further $48 \mathrm{~h}$.

Determination of perforin-positive cell count. The cells to be analyzed were washed once with PBS and fixed with $1 \%$ paraformaldehyde in cold PBS for $20 \mathrm{~min}$. They were centrifuged and the pellet was resuspended in $0.2 \mathrm{ml}$ PBS containing $0.2 \%$ Tween 20 (Panreacsintesis, Spain), and incubated for $20 \mathrm{~min}$ to permeabilize the cell membranes, after which the cells were centrifuged and incubated with fluorochrome-labeled monoclonal antibodies against perforin (perforin-FITC, BD) for $30 \mathrm{~min}$. The cells were washed to remove excess antibody and the number of positive cells was determined by flow cytometry in the lymphocyte region.

Determination of antitumor cytotoxic effect. Cytotoxicity was assessed by analyzing the lactate dehydrogenase (LDH) content in the conditioned medium obtained by co-culture of MNCs stimulated by transfected or control DCs (effector cells) and tumor cells (target cells), using a CytoTox $96^{\circledR}$ Non-Radioactive Cytotoxicity Assay (G1780, Promega, USA). The ratio effector cell: tumor cell was 10:1. Released LDH in culture supernatants is measured with a 30-min coupled enzymatic assay, which results in the conversion of a tetrazolium salt (INT) into a red formazan product. Visible wavelength absorbance data are collected using a standard 96-well plate reader. The amount of color formed is proportional to the number of lysed cells. To convert the concentration of LDH in the supernatant into percentage cytotoxicity, we applied the formula:

$$
\% \text { Cytotoxicity }=\frac{O D(\text { effector }+ \text { target })-O D(\text { effector })-O D(\text { target })}{O D(\text { maximum target lysis })-O D(\text { target })}
$$

To determine the value OD (maximum target lysis), we lysed the tumor cells by Lysis Solution.

Statistical analysis. Statistical data were processed using the Statistica 6.0 program. The Friedman test and Newman-Keuls multiple comparison test were used to detect statistically significant differences. The Shapiro-Wilk test was applied to determine sample normality. The data are presented as the mean and 
standard error for normal distribution, and the median and interquartile range were used for the abnormal distribution.

\section{Results}

Cytotoxic T cells play a central role in the antitumor immune response because they directly lyse tumor cells and produce immunomodulatory cytokines, such as IL-2, TNF- $\alpha$, GM-CSF, and IFN- $\gamma$, which indirectly affect malignant cells. The protective antitumor response involves the killing of tumor cells. Thus, we evaluated MNC cytotoxicity, which is activated via exposure of transfected DCs to autologous tumor cells, by quantifying the cytoplasmic LDH levels of lysed tumor cells. One of the mechanisms through which tumor cell death is triggered is the granule-dependent pathway, which is primarily mediated by perforin, granzyme, and granulysin. Perforin causes pore formation in the target cell membrane and results in cell death $[13$, p. $35 ; 14$ p. 56]. To examine the role of the perforin-dependent mechanism in the lysis of tumor cells, we evaluated the effect of transfected DCs on perforin expression in co-cultures of MNCs from cancer patients.

We demonstrated in patients with breast cancer that the use of lysate-activated dendritic cells or dendritic cells transfected total RNA stimulates the cytotoxic response of the MNC culture that is expressed in an increased cytotoxic activity of MNCs against autologous cells (Figs. 1a and 2). The number of perforin-positive cells increased in the MNC culture stimulated lysate-activated dendritic cells (Fig. 1b).

In patients with NSCLC, DCs primed with an antigen's lysate and DCs transfected with RNA were shown to increase the cytotoxic activity of MNCs against autologous NSCLC cells, compared with the control group (MNC and MNC c-culture with non-primed DCs) (Fig. 3). In the total lymphocyte population after co-culturing lysate-primed DCs and RNA-transfected DCs increase the relative level of perforin-bearing cells in the lymphocyte population compared with control groups (Fig. 4).

In colorectal cancer patients, results indicate that cytotoxicity increased following use of immunogenic lysate-primed DCs, compared with all control groups. The cytotoxicity for the original MNC culture was $11.6 \%$, whereas treatment with lysate-primed DCs increased this to $23.2 \%$ (Fig. 5).

Because tumor cell lysates contain a variety of relevant antigens, a wide range of TAAs is presented to $\mathrm{T}$ cells, inducing a pronounced immune response. By using complete tumor lysates in experimental models and clinical trials, high efficacy and low toxicity have been observed in various cancers, including colorectal $[11$, p. $6445 ; 4$, p. 475]. However, the lysate may contain proteins suppressed the immune response [3, p. 2827] and residual tumor cells in patient organism can change their antigenic composition under the course of treatment that may also complicate the use of the lysate. We demonstrate that the use of dendritic cells to 

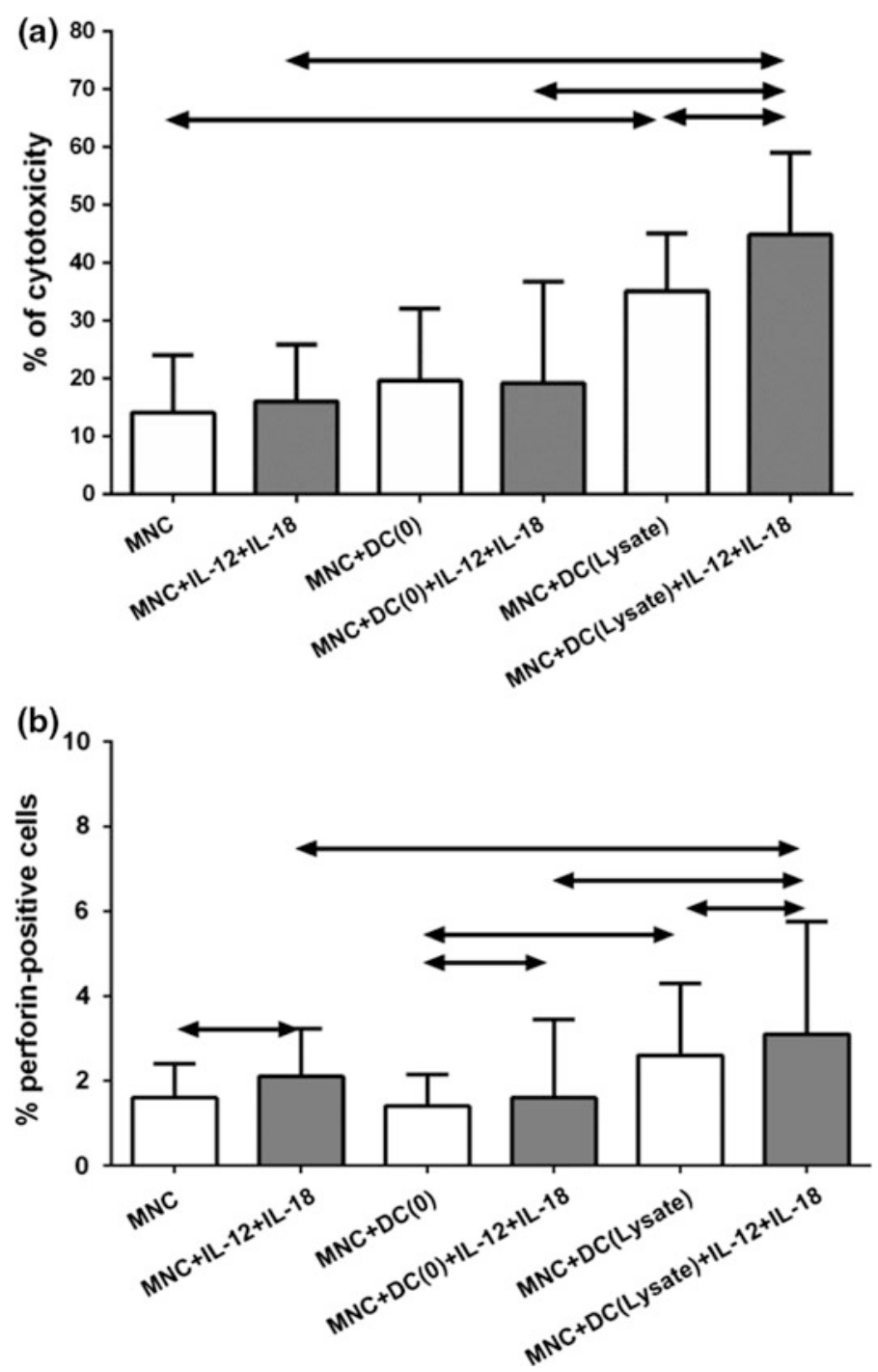

Fig. 1 Cytotoxicity against autologous tumor cells (a) and percentage of perforin-positive cells (b) co-cultured of MNCs and tumor lysate-loaded DCs $(n=20)$ in patients with breast cancer. Data are presented as median $(\mathrm{Me})$ and interquartile range. Arrows indicate statistically significant differences. $(P<0.05)$. MNC - the MNC control culture; MNC + DC $(0)$ - co-culture of MNCs and DCs unloaded with tumor lysate antigens; MNC + DC (Lysate) - co-culture of MNCs and DCs loaded with tumor lysate antigens

prime the lysate's antigens enables efficient activation of the effector functions of immune cells at various cancers. Our findings indicated an elevated cytotoxicity via the perforin pathway, which was corroborated by an increase in the ratio of 


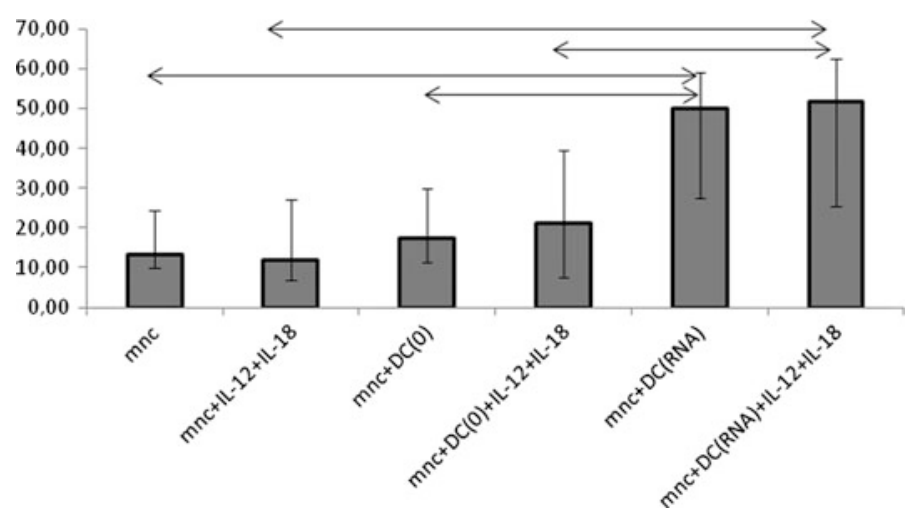

Fig. 2 Cytotoxicity against autologous tumor cells co-cultured of MNCs and tumor DCs, transfected total RNA $(n=16)$ in patients with breast cancer. Data are presented as median $(\mathrm{Me})$ and interquartile range. Arrows indicate statistically significant differences. $(P<0.05)$. MNC the MNC control culture; MNC + DC (0) - co-culture of MNCs and DCs unloaded with tumor antigens; MNC + DC (RNA) - co-culture of MNCs and DCs transfected with total tumor RNA

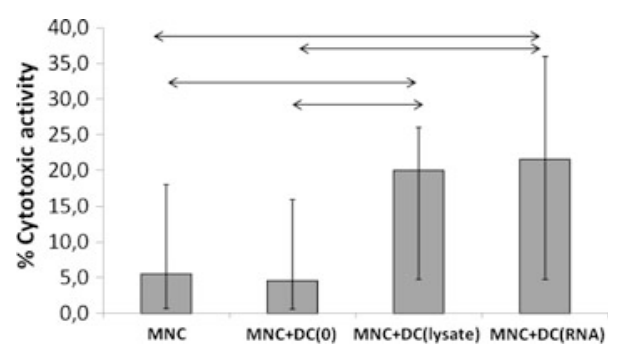

Fig. 3 The effect of antigen-primed DCs on the cytotoxic activity of MNCs against autologous NSCLC cells in vitro, $n=14$. Data are presented as median (Me) and interquartile range. Arrows indicate statistically significant differences. $(P<0.05)$. MNC - the MNC control culture; MNC + DC (0) - co-culture of MNCs and DCs unloaded with tumor antigens; MNC + DC (Lysate) - co-culture of MNCs and DCs loaded with tumor lysate antigens; MNC + DC (RNA) co-culture of MNCs and DCs transfected with total tumor RNA

perforin-positive cells within the total T-cell populations in the group that received RNA-transfected and lysate-primed DCs. Thus, we can speak about the activation of the cytotoxic potential of effector cells, determined by the number of perforin-positive cells. 


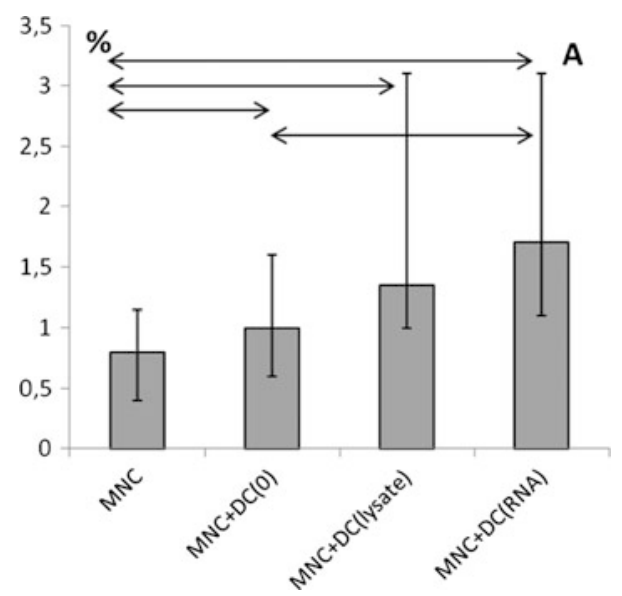

Fig. 4 The effect of antigen-primed DCs on the relative amount of perforin + lymphocytes in the co-culture of MNCs from NSCLC patients, $n=16$. Data are presented as median (Me) and interquartile range. Arrows indicate statistically significant differences. $(P<0.05)$. MNC - the MNC control culture; MNC + DC (0) - co-culture of MNCs and DCs unloaded with tumor antigens; MNC + DC (Lysate) - co-culture of MNCs and DCs loaded with tumor lysate antigens; $\mathrm{MNC}+\mathrm{DC}(\mathrm{RNA})$ - co-culture of MNCs and DCs transfected with total tumor RNA

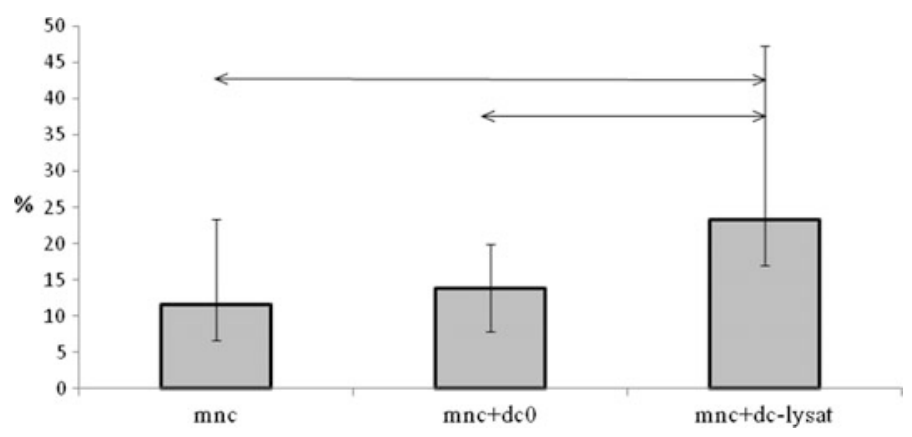

Fig. 5 Cytotoxic activity of colorectal cancer patients MNCs co-cultured with autologous DCs loaded with tumor lysates against autologous tumor cells $(n=17)$. Data are presented as median (Me) and interquartile range. Arrows indicate statistically significant differences. $(P<0.05)$. MNC the MNC control culture; MNC + DC (0) - co-culture of MNCs and DCs unloaded with tumor antigens; MNC + DC (Lysate) - co-culture of MNCs and DCs loaded with tumor lysate antigens

\section{Conclusions}

Currently, there is an intensive development of approaches to induce antitumor immunity using DC-based vaccination technologies. In our opinion, investigates should be primarily concentrated on improving to deliver antigens to DCs to stimulate antitumor immune responses. In addition, the in vitro stimulation of an 
antitumor immune response, which involves the selection and use of various effective stimulators and inhibitors of immunosuppressive molecules to shift the response towards a Th1 response, is also important. In our research, we demonstrated that lysate-primed DC and DC transfected total RNA enhanced MNC antitumor activity, increasing tumor cell death and the percentage of perforin-positive lymphocytes at various cancers. In this regard, we assume that the use of antigens-primed DC in vitro can activate antigen-specific T cells for modulation antitumor response.

Acknowledgements Research is carried out with the financial support of the state represented by the Ministry of Education and Science of the Russian Federation. Agreement no. 14.607.21.0043. Unique identifier: RFMEFI60714X0043.

\section{References}

1. Benencia, F., Sprague, L., McGinty, J., Pate, M., Muccioli, M.: Dendritic cells the tumor microenvironment and the challenges for an effective antitumor vaccination. J. Biomed. Biotechnol. 2012, 1-15 (2012)

2. Boyum, A.: Separation of leukocytes from blood and bone marrow. Scand. J. Clin. Lab. Invest. 21, 97 (1968)

3. Dong, B., Dai, G., Xu, L., Zhang, Y., et al.: Tumor cell lysate induces the immunosuppression and apoptosis of mouse immunocytes. Mol. Med. Rep. 10(6), 2827-2834 (2014)

4. Figdor, C.G., de Vries, I.J., Lesterhuis, W.J., Melief, C.J.: Dendritic cell immunotherapy: Mapping the way. Nature Med. 10(5), 475-480 (2004)

5. Fricke, I., Gabrilovich, D.I.: Dendritic cells and tumor microenvironment: A dangerous liaison. Immunol. Invest. 35, 459-483 (2006)

6. Janikashvili, N., Larmonier, N., Katsanis, E.: Personalized dendritic cell-based tumor immunotherapy. Immunotherapy 2(1), 57-61 (2010)

7. Liu, L.N., Shivakumar, R., Allen, C., Fratantoni, J.C.: Delivery of whole tumor lysate into dendritic cells for cancer vaccination. In: Li, S. (eds.) Electroporation protocols, Methods Mol. Biol. vol. 423, pp. 139-153. (2008)

8. Marchini, C., Kalogris, C., Garulli, C.: Tailoring DNA vaccines: designing strategies against HER2-positive cancers. Front Oncol. 3, 122 (2013)

9. McNamara, M.A., Nair, S.K., Holl, E.K.: RNA-based vaccines in cancer immunotherapy. J. Immunol. Res. 2015, (2015)

10. Peres, Lde P., da Luz, F.A., Pultz Bdos, A., Brígido, P.C., de Araújo, R.A., Goulart, L.R., Silva, M.J.: Peptide vaccines in breast cancer: the immunological basis for clinical response. Biotechnol. Adv. 33(8), pp. 1868-77. (2015)

11. Schnurr, M., Galambos, P., Scholz, C., et al.: Tumor cell lysate-pulsed human dendritic cells induce a T-cell response against pancreatic carcinoma cells: An in vitro model for the assessment of tumor vaccines. Cancer Res. 61, pp. 6445-50. (2001)

12. Ugel, S., Facciponte, J.G., De, Sanctis F., Facciabene, A.: Targeting tumor vasculature: Expanding the potential of DNA cancer vaccines. Cancer Immunol. Immunother. 64(10), 1339-1348 (2015)

13. Voskoboinik, I., Dunstone, M.A., Baran, K., Whisstock, J.C., Trapani, J.A.: Perforin: Structure, function, and role in human immunopathology. Immunol Rev. 235(1), pp. 35-54. (2010)

14. Zhou, F.: Perforin: More than just a pore-forming protein. Int. Rev. Immunol. 29(1), pp. 5676 (2010) 
Open Access This chapter is licensed under the terms of the Creative Commons Attribution 4.0 International License (http://creativecommons.org/licenses/by/4.0/), which permits use, sharing, adaptation, distribution and reproduction in any medium or format, as long as you give appropriate credit to the original author(s) and the source, provide a link to the Creative Commons license and indicate if changes were made.

The images or other third party material in this chapter are included in the chapter's Creative Commons license, unless indicated otherwise in a credit line to the material. If material is not included in the chapter's Creative Commons license and your intended use is not permitted by statutory regulation or exceeds the permitted use, you will need to obtain permission directly from the copyright holder.

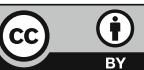

